\title{
Agarelektrophoretische Studien zur Charakterisierung von menschlichem Milzextrakt
}

\author{
Von \\ F. Scheiffarth, H. Götz und D. HofFmanN \\ Als der Medizinischen Universitätsklinik Erlangen-Nïrnberg (Direktor: ̈̈rof. Dr. med. N. Henning)
}

(Der Schriftleitung zugegangen am 1. Oktober 1963.)

\begin{abstract}
Es wurden 15 Milzextrakte agarelektrophoretisch, färberisch mit Nachweismethoden fermentaktiver Kompoñèñten, sowie immunologisch untersucht mit dem Ziel einer Charakterisierung der Proteinkomponenten yon Milzextrakt. Als Bezugsystem für diese Untersuchungen wurde menschliches Normalserum verwendet. Zur geeriaueren Lokalisation einzelner Komponenten wurden unter anderem hämolytische Seren sowie Seren mit Paraproteinen von bestimmten Typen mitverwendet. Es kann festgestellt werden, daß Milzextrakt sich vom Humanserum in qualitativer und quantitativer Hinsicht wesentlich unterscheidet. Es muß aber auch festgestellt werden, daß einzelne Komponenten mit Fraktionen des menschlichen Serums prinzipiell vergleichbar sind, auch wenn hier quantitative Differenzen bestehen. Die Ergebnisse wurden im Sinne der Charaktẹrisier̦ung der Proteinsysteme von Milzextrakten diskutiert.
\end{abstract}

\begin{abstract}
In order to characterise the protein components of spleen extract, 15 extracts were studied by agar electrophoresis, by colour reactions for enzymes and by immunology. Normal, human serum was used as a reference system. For a more exact localisation of individual components, haemolytic sera and sera with particular types of paraproteins were also used. It can be shown that spleen extract is qualitatively and quantitatively essentially different from human serum. Some individual fractions must, however, be comparable with fractions from human serum, even if there are quantitative differences. The use of these results for the characterisation of the proteins of spleen extract is discussed.
\end{abstract}

In vorliegender Arbeit wurden in eigener Versuchsanordnung Milzextrakte im Vergleich zu einem Normalserum agarelektrophoretisch, immunoelektrophoretisch und durch Färbe- und Fermentreaktionen analysiert. Diese Untersuchungen sollten der Charakterisierung der verschiedenen in der Milz nachweisbaren Eiweißkomponenten dienen, wobei sowohl die Frage einer Identität von Milz- und Serumproteinen als auch die Frage der Lokalisation und Differenzierung verschiedener Fermente durch Nachweis ihrer Aktivität erörtert werden sollte. Wie in früheren Untersuchungen $(1,2,3,4)$ an menschlichen Organextrakten gezeigt werden konnte, eignen sich hierfür die Kombinationstechniken der Agarelektrophorese in besonderem Maße.

\section{Versuche}

\section{Untersuchungsgut}

Es wurden insgesamt 15 verschiedene Milzorgane von der Leiche analysiert ${ }^{1}$ ). Die Organe kamen nicht später als 24 Stunden post mortem zur Verarbeitung; es handelte sich ausschließlich um histologisch unveränderte Organe. Hinsichtlich des Alters und des Geschlechts der Verstorbenen wurde keine Auswahl getroffen.

Versuchsanordnung

Von jedem der Organe wurden Extrakte hergestellt und in folgender Weise analysiert:

a) Einfache Elektrophorese im Agar-Gel mit Protein-, Kohlenhydrat- und Lipoidfärbung.

1) Die Organpräparate verdanken wir dem Pathologischen Institut der Universität Erlangen-Nürnberg (Direktor: Prof. Dr. E. MüLLER). b) Nachweis der oxydaseaktiven Coeruloplasminfraktionen.

c) Darstellung der Komponenten mit alkalischer Phosphataseaktivität.

d) Reaktion auf Diastaseaktivität.

e) Immunoelektrophoretische Analyse mit Antihumanserum.

Für jeden Analysensatz wurde das Serum eines Gesunden ver. gleichsweise mit angesetzt.

$\cdots$

Methodik

a) Aufbereitung der Organe $(1,3,4)$

Fettsubstanz, Bindegewebe einschließlich Kapsel wurden weitgẹend abpräpariert. Die Organe wurden zerkleinert und zur Entblutung 4 Stdn. im fließenden Wasser gespült. Diese Gewebsstücke wurden weiter in kleine Würfel geschnitten und unter $\mathrm{Zu}$ gabe von Aqua dest. im Multimix (10 Min.) zertrümmert.

Der gewonnene Organbrei wurde $30 \mathrm{Min}$. bei $3000 \mathrm{U} / \mathrm{Min}$. bei Zimmertemperatur zentrifugiert. Der Uberstand des Zentrifugats wurde abpipettiert, zur Einengung gegen 20-proz. Kollidon in Dialysierschläuche ${ }^{2}$ ) gefüllt und über zwei Tage im Kühlschrank bei $+4^{\circ}$ auf eine mindestens $2-3$-proz. Eiweißlösung eingeengt. Auch die Bodenschicht, die schwer löslich war, wurde bei 6 verschiedenen Milzorganen weiterverarbeitet, und zwar wurde sie mit physiol. Kochsalzlösung versetzt (2 Teile Organbrei auf 1 Teil physiol. Kochsalzlösung). Dann wurde dieser Organextrakt mit einem „Ultra-Turrax “'-Gerät ${ }^{3}$ ) zu einem feinen Brei zermahlen (5 Min.) und anschließend bei Zimmertemperatur $45 \mathrm{Min}$. bei 4000 U/Min. zentrifugiert. Die überstehende Flüssigkeit wurde abpipettiert, zur Einengung gegen 20-proz. Kollidon wiederum in Dialysierschläuche gefüllt und auch zwei Tage bei $+4^{\circ}$ belassen. Der Rückstand wurde diesmal verworfen. Die eingeengten Extrakte wurden zur weiteren Analyse verwendet.

2) Dialysierschläuche der Membranfiltergesellschaft, Göttingen:

3) „Ultra-Turrax" der Fa. Janke \& Kunkel KG., Staufen i. Br. 
b) Einfacho Elcktrophorese im Agar-Gel mit Färbemetboden und Fernsentrcaktionen

Bei jedem Analysensatz wurden die Extrakte der Milz jeweils im Vergleich mit einem Humanserum im Agar-Gel elektrophoretisch aufgetrennt. Methodische Einzelheiten dieser Elektrophorese sind dem einschlägigen Schrifttum zu entnehmen $(5,6,7,8,9,10)$. Zur Charakterisierung der einzelnen Eiweißkomponenten wurden folgende Färbungen und Fermentreaktionen angewandt:

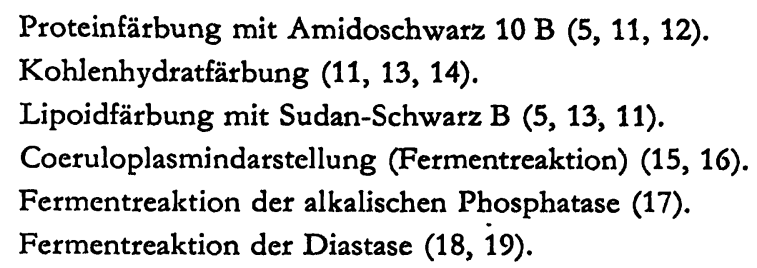

c) Immunoelcktrophorese $(6,7,8,10)$

Die einzelnen Organextrakte sowie das Vergleichsserum eines Gesunden wurden weiterhin mit Hilfe der Immunoelektrophorese nach Grabar und Williams analysiert. Als Immunserum wurde ein Humanserum-Antiserum ${ }^{1}$ ) verwendet. Dieses wurde im Verhältnis $1: 1,3$ mit physiol. Kochsalzlösung verdünnt. Weitere Einzelheiten hierzu sind dem einschlägigen Schrifttum zu entnehmen.

\section{Ergebnisse}

\section{Einfache Agarelektrophorese}

Bei Färbung eines agarelektrophoretisch getrennten Milzextraktes mit Amidoschwarz $10 \mathrm{~B}$ lassen sich insgesamt 8-9 Komponenten abgrenzen. Unter ihnen imponieren im wesentlichen zwei relativ stark gefärbte Fraktionen weit anodisch wandernd, eine weitere unmittelbar anodisch vom Auftragstrich entfernt sowie eine besonders stark gefärbte und breit verlaufende Komponente im Bereich kathodisch von der Auftragstelle entfernt. Außerdem lassen sich weit anodisch vor den Hauptkomponenten 1-2 Vorfraktionen einerseits sowie zwei relativ schwach gefärbte aber scharf abgrenzbare Komponenten jenseits des Auftragstriches kathodisch wandernd nachweisen.

Vergleicht man einen Milzextrakt mit einem Humanserum, so fällt auf, daß Milzextrakt insgesamt einen weitaus größeren Trennbereich aufweist als Humanserum. Es lassen sich außerdem hinsichtlich der quantitativen und auch der qualitativen Verteilung der einzelnen Eiweißkomponenten deutliche Unterschiede nachweisen. Die Zuordnung der verschiedenen Komponenten zu Eiweißkörpern des menschlichen Serums soll in der Diskussion abgehandelt werden.

Bei Färbung eines agarelektrophoretisch aufgetrennten Milzextraktes mit Schiffschem Reagens lassen sich vor allem die schnell wandernden Fraktionen deutlich darstellen, ebenso aber auch die Komponente im Bereich kathodisch von der Auftragstelle. - Bei Färbung des Milzextraktes mit Sudanschwarz B zum Nachweis der Lipoproteide findet sich lediglich in einem kleinen Bezirk anodisch vom Auftragstrich eine schwache Anfärbbarkeit, die jedoch nicht bei allen Extrakten reproduzierbar war.

1) Anti-Normalserum, IEP Antiserum, Hyland Laboratories, Los Angeles Calif., USA.
Prüft man die agarelektrophoretisch getrennten Milzextrakte auf ihre Oxy'daseaktivität mit Färbung durch pPhenylendiamin, so läßt sich in allen Fällen eine unterschiedlich intensive, jedoch einwandfrei nachweisbare Reaktion im $\alpha$-Bereich, d.h. anodisch vom Auftragstrich, aufzeigen.

Alkaliscle Phosphatase findet sich auf Grund der typischen Fermentreaktionen in einem Bereich unmittelbar um den Auftragstrich, und zwar sowohl anodisch wie kathodisch. Dies ist in allen Präparaten einwandfrei reproduzierbar.

Diastaseaktivität läßt sich unter Zuhilfenahme der vorliegenden Nachweismethode bei den hier untersuchten Milzextrakten nicht nachweisen.

Zusätzliche Studien von verschiedenen Extraktsubstraten, d. h. von elektrophoretisch getrenntem Bodensatz und Überstand (siehe Aufbereitungsmethode) ergeben sich einige Unterschiedlichkeiten der Art, daß die untersuchten Überstände im wesentlichen weitaus bessere Resultate aufweisen als der Bodensatz. Einen Unterschied bietet lediglich die Reaktion auf Oxydaseaktivität; hier findet sich eine deutlichere Reaktion im Bodensatz, während der untersuchte Überstand zwar eine adäquate, jedoch eine quantitativ geringere Anreicherung von Oxydaseaktivität aufweist.

\section{Immunoelektrophorese}

In vorliegender Arbeit wurde jeweils der agarelektrophoretisch aufgetrennte Organextrakt lediglich mit einem Antihumanserum geprüft. Hierbei ergaben sich eine Reihe von Präzipitationslinien, die teilweise mit Komponenten des menschlichen Serums vergleichbar waren, die jedoch unterschiedlich intensiv ausgefallen waren. Allerdings ließen sich außerdem Komponenten nachweisen, die offentsichtlich im menschlichen Serum nicht aufzuzeigen sind. Eine Beschreibung der einzelnen Präzipitationslinien an dieser Stelle erübrigt sich, da in der Diskussion auf die einzelnen Eiweißsysteme im Vergleich mit menschlichem Serum näher einzugehen bleibt.

Insgesamt ist festzustellen, daß die Ergebnisse der verschiedenen Extrakte im großen und ganzen gute Übereinstimmung zeigten, d. h., daß die vorgenannten Aussagen im wesentlichen reproduzierbar waren.

\section{Diskussion}

In vorliegenden Studien dienen der Charakterisierung von Proteinsystemen der Milz im wesentlichen folgende Faktoren:

1. Die elektrophoretische Mobilität im Agar-Gel im Vergleich mit bekannten Eiweißkörpern des menschlichen Serums.

2. Färbemethoden zum Nachweis von Proteinen, Glykoproteiden sowie Lipoproteiden, sowie Nachweismethoden zur Aufklärung fermentaktiver Komponenten.

3. Die Intensität von Färbungen für quantitative Relationen. 


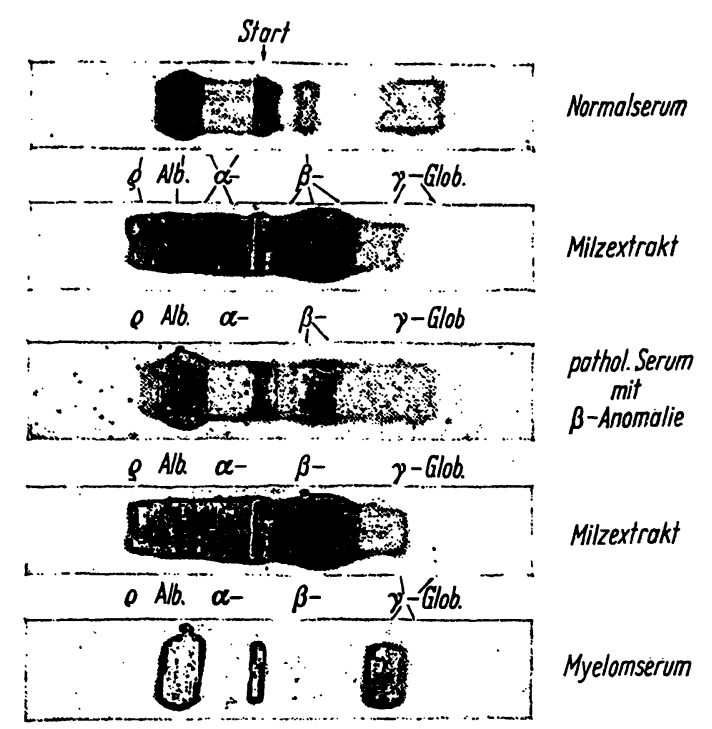

Abb. 1

Proteingefärbte Agarelektrophoresen von Milzextrakten und menschlichen Seren

4. Immunologische Kriterien nach Reaktion mit einem Antihuman-Immunserum.

Wie in früheren Arbeiten bereits erwähnt (1-4), werden in den folgenden Beispielen die mit menschlichen Serumproteinen vergleichbaren Komponenten des Milzextraktes entsprechend bezeichnet, ohne daß damit die absolute Identität solcher Fraktionen unterstellt werden soll.

Vergleicht man einen in Agar gewanderten und proteingefärbten Milzextrakt mit einem unter gleichen Bedingungen gewanderten menschlichen Serum (Abb. 1), so fällt auf, daß Milzextrakt eine deutlich größere Trennstrecke einnimmt als Humanserum, wobei die Komponenten in anodischer Richtung weiter wandern als diejenigen jenseits der Auftragstelle. Außerdem zeigen Milzextrakt und Serum quantitativ und qualitativ unterschiedliche Komponenten. Auffällig sind Fraktionen im Präalbuminbereich, die sich im Serum in diesem Maße nicht finden. Außerdem zeigt sich in der Zone der $\alpha_{2}$-Globuline eine grünlich gefärbte Komponente, die wohl einer hämoglobinreichen Fraktion entsprechen dürfte. Desgleichen läßt sich im $\beta$-Bereich eine derartige Fraktion nachweisen, die mit hoher Wahrscheinlichkeit dem hämoglobintragenden Protein entspricht. Vergleicht man nämlich ein hämolytisches Serum mit einem Milzextrakt, so findet sich an gleicher Stelle, d. h. im Bereich der $\beta_{1}$-Globuline eine Fraktion, die dem Hämoglobin entspricht. Weiterhin auffällig ist am Milzextrakt die regelmäßig reproduzierbare Aufteilung des $\beta / \gamma$-Bereiches in mindestens zwei Komponenten, wobei die eine, die einem $\beta_{2}$-Protein entsprechen dürfte, regelmäßig stärker ausgeprägt ist als die andere Fraktion, die im Vergleich zum menschlichen Serum als $\gamma$-Globulin definiert werden kann. Zwischen $\beta_{1}$ - und $\beta_{2}$-Globulinen läßt sich, allerdings nicht in allen Analysen, eine schwache zusätzliche Fraktion nachweisen. Im

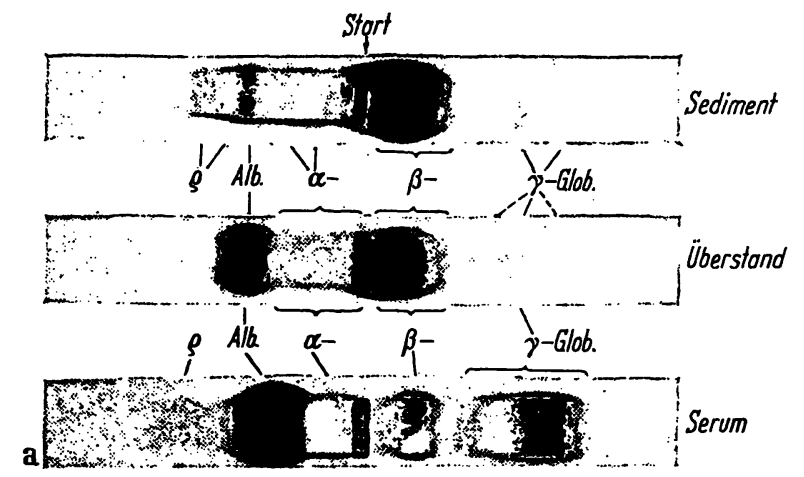

Abb. 2a

Gegenüberstellung zweier verschiedener Aufbereitungsphasen von Milzextrakt nach agarelektrophoretischer Trennung und Proteinfärbung im Vergleich zu einem menschlichen Normalserum

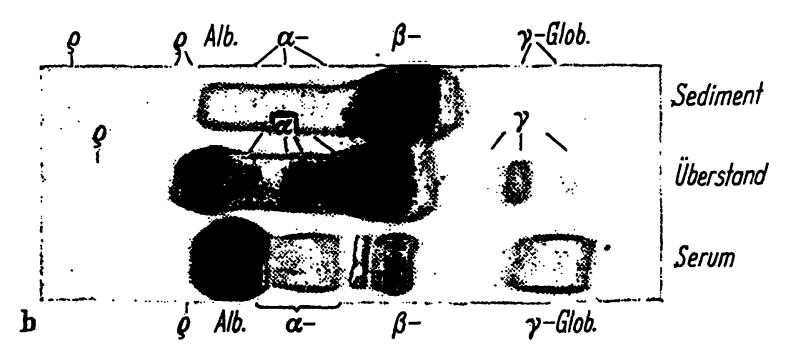

Abb. $2 b$

Agarelektrophorese bei optimaler Proteinausbeute des Milzextraktes

anodisch wandernden Teil lassen sich außer den bereits erwähnten Fraktionen in Einzelfällen weitere Unterfraktionen aufzeigen; dies einerseits im Albuminvorbereich, andererseits in der Zone zwischen Albumin, $\alpha_{1}$-Globulin und $\alpha_{2}$-Globulin (Abb. 2).

Vergleicht man darüber hinaus die in den Ergebnissen angeführten verschiedenen Aufbereitungsstufen, d. h. Überstand des Milzextraktes sowie Sediment, so fällt in allen Proben auf, daß der Überstand die größere Proteinausbeute aufweist als das Sediment und daß gesetzmäßig gewisse Unterschiede zwischen der einen und der anderen Phase bestehen. Besonders auffällig ist dabei die Tatsache, daß im Uberstand eine Komponente mit der Wanderungsgeschwindigkeit etwa eines menschlichen Albumins vorherrscht und dạ $\beta$ auch die zwischen $\beta_{1}$ und $\gamma$ gelegene Fraktion deutlich nachweisbar ist. Im Sediment dagegen findet sich die dem Albumin vergleichbare Komponente in nur geringer Konzentration bzw. sie wird durch ein schnell wanderndes $\alpha$-Globulin ersetzt. Außerdem zeigen sich meist zwei weit vor dem Albumin wandernde Proteine. Schließlich wird die $\beta_{2}$-Fraktion im Sediment erheblich vermindert angetroffen.

Kupfer bzw. oxydaseaktive Fraktionen werden im Milzextrakt einwandfrei nachgewiesen. Sie finden sich im $\alpha$-Bereich, wobei bei Untersuchung des überstehenden Substrates der Kupfergehalt offentsichtlich geringer ist als bei Untersuchung der Sedimente. Dies mag unter Umständen mit Elementen der Milz zusammenhängen, die vorwiegend dem mesenchymalen, $d . h$. bindegewe- 


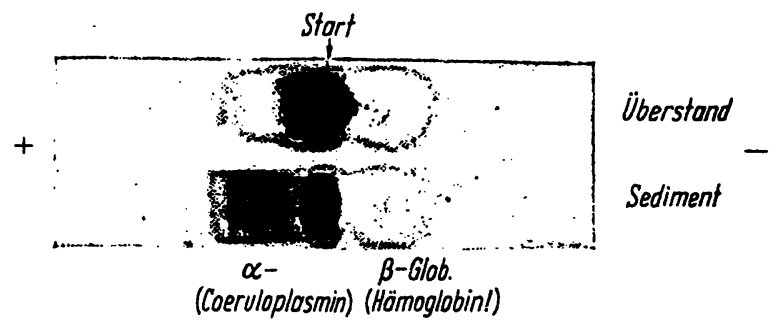

Abb. 3

Coeruloplasmindarstellung (Oxydaseaktivität) in Milzextrakten. Oben Uberstand, unten Sediment

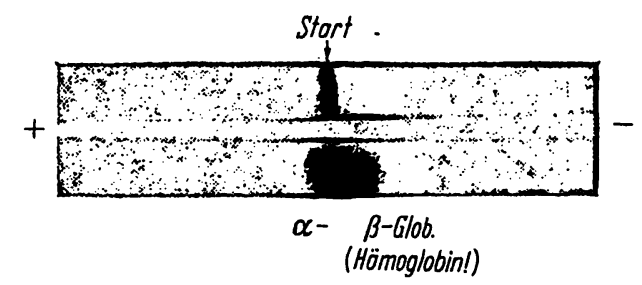

Abb. 4

Darstellung der alkalischen Phosphatasen in Milzextrakten

bigen Anteil dieses Organes entsprechen dürften (Abb.3). Alkalische Phosphatase wird ebenfalls, und zwar offenbar in hohem Maße, im Milzextrakt nachgewiesen. Die Aktivität findet sich vorwiegend im $\alpha_{2}$-Bereich, aber auch im $\beta_{1}$-Bereich, $d . h$. anodisch und kathodisch um die Auftragstelle. Im $\beta$-Bereich wird die Farbreaktionteilweise von Hämoglobin überlagert (Abb. 4).

Eine Gewebsdiastase konnte, wie bereits erwähnt, nicht aufgezeigt werden. Daraus ist möglicherweise der Schluß zu ziehen, daß tatsächlich in Milzextrakten überhaupt keine Diastase vorliegt. Es muß allerdings auch bedacht werden, daß die hier verwandte Untersuchungsmethode zum Nachweis einer Diastaseaktivität geringste Mengen von Diastase, wie sie unter normalen Bedingungen z. B. im menschlichen Serum vorhanden sind, nicht erfaßt. In der Tat werden z. B. die geringen Aktivitäten des Normalserums mit der Agardiastasemethode nicht erfaßt.

Gemessen an entsprechenden Farbreaktionen handelt es sich bei den schnell wandernden Fraktionen des $\alpha$ Bereiches um Glykoproteide. Glykoproteide finden sich jedoch auch im Bereich des hämoglobinhaltigen $\beta_{1^{-}}$ Proteins (Abb. 5). Lipoproteide werden in den hier untersuchten Milzextrakten weitgehend vermißt.

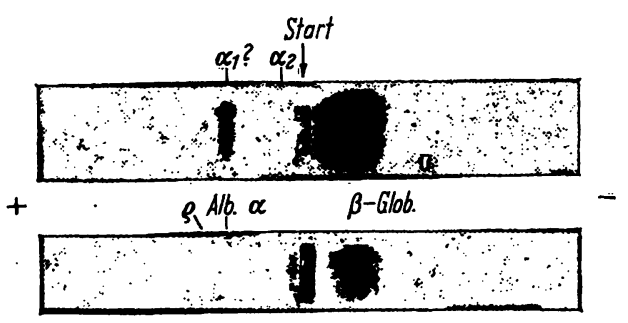

Abb. 5

Kohlenhydratgefärbte Komponenten (Glykoproteide) von Milzextrakten. Oben Uberstand, unten Sediment
Die Problematik der Studien von Organextrakten mit Hilfe agarelektrophoretischer und färberischer Methoden liegt in verschiedenen Komponenten: Erstens in der Tatsache, daß unter den Umständen der Aufbereitung eines Organes Proteinsysteme ihrer ursprünglichen Eigenschaften verlustig werden können. Die Problematik liegt zweitens darin, daß der Vergleich mit einem bekannten bzw. standardisierten Eiweißgemisch vom Typ des menschlichen Serums nur eine relative Aussage ermöglicht. Drittens muß gerade bei Milzextrakten, trotz sorgfältiger Wässerung, mit einem hohen Blutreichtum gerechnet werden. Immerhin kann jedoch zufolge der hier angeführten Ergebnisse festgestellt werden, daß Milzextrakt ein für sich typisches Eiweißspektrum aufweist, das durch die Länge der Trennstrecke, die reichlich anodisch wandernden Fraktionen, die hämoglobinhaltigen Komponenten und nicht zuletzt die Unterteilung des $\beta / \gamma$-Bereiches in mehrere Komponenten charakterisiert wird. Der Hämoglobinreichtum des Extraktes ist erklärbar durch die physiologische Funktion der Milz als Blutmauserungsorgan. Die Tatsache, da $\beta$ zwei Bereiche, nämlich der $\alpha_{2}$-Bereich wie auch der $\beta_{1}$-Bereich mit Hämoglobin beladen sind, mag zu der Deutung verleiten, daß hier verschiedene Hämoglobinarten differenzierbar sind. Vielleicht handelt es sich einerseits um das Hämoglobin gewanderter Erythrozyten, andererseits um gelöstes bzw. an $\beta_{1}$-Protein gebundenes Hämoglobin.

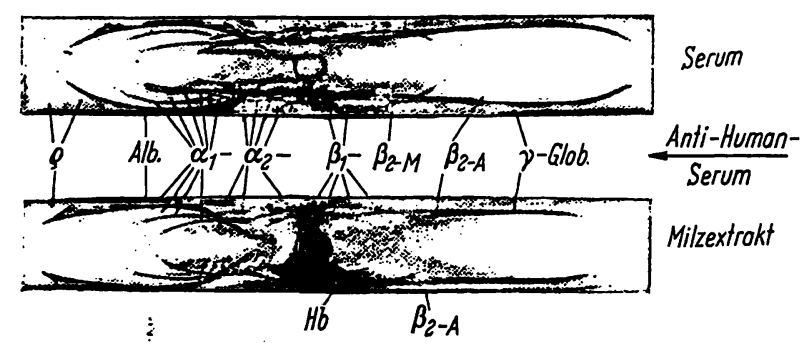

Abb. 6

Immunoelektrophoresen von menschlichem Serum (oben) und Milzextrakt (unten)

Bei Beurteilung der immunoelektrophoretischen Befunde aufgetrennter Milzextrakte im Vergleich zu einem Normalserum lasșen sich einerseits Linien nachweisen, die im wesentlichen den bekannten Komponenten des menschlichen Serums entsprechen, andererseits aber auch solche, die kein adäquates Phänomen mit menschlichen Serumproteinen zeigen. Die auffälligsten Unterschiede gegenüber dem Serum finden sich im $\alpha_{2}$ und $\beta$-Bereich. Sicher handelt es sich hier um Linien, die einer Haptoglobin bzw. Hämoglobinkomponente entsprechen. Es findet sich sicher auch ein Proteinsystem, das dem Siderophilin zuzuschreiben ist. Es läßt sich ein $\beta_{2}$-Globulin, das dem $\beta_{2}$-A-Globulin entsprechen dürfte, nachweisen, und es findet sich eine Fraktion, die ihrer Ausdehnung nach dem $\gamma$-Globulin des menschlichen Serums entsprechen kann. Gemessen an der Intensität der einzelnen Linien, fällt die Albuminfraktion im Sinne des Antigenüberschußphänomens auf und es läßt sich 
andererseits $\operatorname{der} \beta$ - und $\gamma$-Bereich als verhältnismäßig quantitativ gering aufzeigen.

Nähere Aussagen ohne Absorption bzw. Identitätsreaktionen mit isolierten Komponenten wären hier spekulativ. Es müßten sich biochemische bzw. organ- analytische Untersuchungen anschließen um die genauere Aufklärung eines Milzextraktes zu ermöglichen und um die Frage zu klären, inwieweit spezifische Identitätsreaktionen zwischen Serumeiweißkörpern und Organeiweißkörpern vorliegen.

\title{
Literatur
}

1. Götz, H. und F. Scheiffarth, Klin. Wschr. 41, 587 (1963). 2. Götz, H., F. Scheiffarth und I. Dübeler, Gastroenterologia, Basel 98, 30 (1962). - 3. Scheiffarth, F., H. Götz und G. Schernthaner, Clin. chim. Acta (Amsterdam) 6, 481 (1961). 4. Scheiffarth, F., H. Götz und G. Thuma, Clin. chim. Acta (Amsterdam) 7, 355 (1962). - 5. Götz, H., G. Berg und F. SCheIfFARTH, Zschr. Immunit.forsch., Jena 114, 72 (1962). 6. Grabar, P., Bull. Soc. Chim. biol. 36, 65 (1954); Dtsch. med. Wschr. 79, 1916 (1954); Zbl. Bakteriol. 164, 15 (1955). - 7. Grabar, P. und P. Burtin, Analyse immuno-électrophorétique. Application avec liquides biologiques humains. Masson \& Cie., Paris (1960). - 8. Grabar, P. und C. A. Williams, Biochim. biophysica Acta (Amsterdam) 10, 193 (1953); 17, 67 (1955). 9. Scheidegger, J. J., Internat. Arch. Allergy 7, 103 (1955). 10. Williams, C. A. und P. Grabar, J. Immunol., Baltimote 74,
158 (1955). - 11. Uriel, J. und P. Grabar, Ann. Inst. Pasteur, Paris 90, 429 (1956). - 12. URIEL, J. und J. J. Scheideggrr, Bull. Soc. Chim. biol. 37, 165 (1955). - 13. Scheifrarth, F., H. GötZ und H. KNopfF, Acta haemat. 26, 169 (1961). - 14. Uriel, J., P. Grabar und Ch. Wunderly, Clin. chim. Acta (Amsterdam) 2, 35 (1957). - 15. Scheiffarth, F., H. Götz und F. CzAGANY, Med. Welt 28, 1449 (1961). - 16. URIEL, J., Bull. Soc. Chim. biol. 39, Suppl. I, 105 (1957); Clin. chim. Acta (Amsterdam) 3, 17 (1958); 3, 384 (1958). - 17. ScheIfFARTH, F., H. Warnatz und H. Götz, Med. Welt 43, 2216 (1961). - 18. HeInkel, K., in: N. Henning, Klinische Laboratoriumsdiagnostik, 2. Aufl., S. $431 \mathrm{ff}$., Urban \& Schwarzenberg, München-Berlin (1960). - 19. ScheIfFARTh, F. und H. Götz, Klin. Wschr. 42, 130 (1964).
Professor Dr. F. Scheiffarth Medizinische Universitätsklinik 852 Erlangen, Krankenhausstr. 12

\section{Zur Bestimmung des Pregnandiols im Harn nach KLOPPER, MICHIE und BROwN ${ }^{1}$ )}

Von

\author{
G. E. HALDER
}

\author{
Aus dem Laboratorium der Frauenklinik des Städt. Krankenbauses im Friedricbshain \\ (Seiner Zeit Dirig. Arat: Prof. Dr. Dr. W. Pschyrembel)
}

(Der Schriftleitung zugegangen am 12. November 1963)

\begin{abstract}
In Anbetracht der weiten Verbreitung, die die KLoppersche Methode zur quantitativen Bestimmung des Pregnandiols im Harn gefunden hat, wird über eigene Erfahrungen bei der routinemäßigen Durchführung dieser Bestimmungen berichtet. Neben einigen Modifikationen wird insbesondere die Anwendung der AlLENschen Korrektur bei der Messung der Pregnandiol-Farblösungen empfohlen. An 66 Harnen wurde sowohl die monochromatische Messung bei $430 \mathrm{~m} \mu$ wie die Allensche Korrektur bei 380, 420 und $460 \mathrm{~m} \mu$ durchgeführt. Hierbei werden gegenüber der Originalmethode im Mittel um 28\% niedrigere Pregnandiol-Ausscheidungen gemessen. Eine Abkürzung der 17-stdg. Entwicklung des Pregnandiol-Farbkomplexes ist nicht möglich.

In view of the widespread use of KLOPPER's quantitativ determination of pregnandiol in urine, results from the routine use of the method in this laboratory are reported. Some modifications and especially the use of ALLEN's correction in measuring the pregnandiol colour are recommended. Both monochromatic measurements at $430 \mathrm{~m} \mu$ and AlLEN's correction at 380,420 and $460 \mathrm{~m} \mu$ were performed on 66 urines. The measured pregnandiol excretions were on an average $28 \%$ lower than shown by the original method. The 17 hours development time of the pregnandiolcolour complex could not be shortened.
\end{abstract}

Die von Klopper, Michie und Brown (1) im Jahre 1955 publizierte Methode zur quantitativen Bestimmung des Pregnandiols im Urin erwies sich als das

1) Diese Arbeit wurde in den Jahren 1960/61 durchgeführt. z. Z. genaueste und empfindlichste Verfahren. Sie löste ältere Methoden, insbesondere die von VenNing angegebene ab $(2,3)$. Mit dieseñ Verfahren war eine Erfassung geringer Pregnandiolgehalte nur mögliç, gesammelt und aufgearbeitet wurden $(4,5)$. Die von 